\title{
Solving practical economic load dispatch problem using crow search algorithm
}

\author{
Shaimaa R. Spea \\ Electrical Engineering Department, Faculty of Engineering, Menoufiya University, Egypt
}

\begin{tabular}{l} 
Article Info \\
\hline Article history: \\
Received Sep 25, 2019 \\
Revised Jan 18, 2020 \\
Accepted Feb 2, 2020 \\
\hline
\end{tabular}

Keywords:

Crow search algorithm Economic load dispatch Multiple fuel options Valve-point effects

\begin{abstract}
The practical economic load dispatch problem is a non-convex, non-smooth, and non-linear optimization problem due to including practical considerations such as valve-point loading effects and multiple fuel options. An optimization algorithm named crow search algorithm is proposed in this paper to solve the practical non-convex economic load dispatch problem. Three cases with different economic load dispatch configurations are studied. The simulation results and statistical analysis show the efficiency of the proposed crow search algorithm. Also, the simulation results are compared to the other reported algorithms. The comparison of results confirms the high-quality solutions and the effectiveness of the proposed algorithm for solving the non-convex practical economic load dispatch problem.
\end{abstract}

Copyright $\odot 2020$ Institute of Advanced Engineering and Science. All rights reserved.

\section{Corresponding Author:}

Shaimaa Rabah Spea,

Department of Electrical Engineering,

Faculty of Engineering, Menoufiya University,

Shebin El-kom, Egypt.

Email: shi_spea@yahoo.com

\section{INTRODUCTION}

Economic load dispatch (ELD) is an essential optimization task in the power system. It represents a basic problem in the power system operation, which objects to achieve the minimum cost of energy requirements while satisfying all the unit and system constraints [1]. In the simplest formulation of the ELD problem, the fuel cost function of the generation unit is represented by a quadratic function, and the valve point loading effects (VPL) are ignored, which have the advantages of being smooth and convex. These advantages increase the number of optimization methods that can easily implement to find the solution for the ELD problem. In practical operating conditions of the power system, many thermal generation units are supplied with different sources of fuel, such as natural gas, oil, and coal. It is necessary to find the most economical fuel to be used in these units [2]. To model the multiple fuel options (MFO), the piecewise quadratic function is used for the representation of fuel cost function [3]. The practical ELD with VPL and MFO is a non-convex, non-continuous, and non-differentiable optimization problem with many equality and inequality constraints, which makes it very difficult to find the optimal solution of this problem [4].

For its importance, many researchers try to solve the ELD problem using a verity of conventional and non-conventional methods. The conventional methods such as Quadratic Programming [5] and Linear Programming [6] often fail to obtain the best solutions to the non-convex problems as they assumed that the functions are smooth and convex. Also, the convergence of these methods depends on the initial points, and they are easy to converge into the local optimal solution. Thus many of the conventional methods are not efficient to find the solution of the ELD problem, especially when the practical conditions are considered. To overcome the limitations of the classical methods, various Evolutionary Algorithms (EAs) have been implemented to solve the ELD problem such as Social Spider Algorithm (SSA) [4], Genetic Algorithm (GA) [7], Chaotic Bat Algorithm (CBA) [8], Elephant Herding Optimization (EHO) [9], 
Backtracking Search Algorithm (BSA) [10], Moth Flame Algorithm (MFA) [11], etc. Most of these algorithms have fast convergence characteristics and high precision. So they can deal more effectively and robustly with practical and large-scale problems. In this paper, a Crow Search Algorithm (CSA) is proposed to solve the non-convex practical ELD problem considering VPL and MFO. The proposed CSA is tested on 10-unit test system, large scale test systems with 30, 60, and 100 units, and very large-scale test systems with 500, 1500, 2000, and 2500 units. The simulation results are compared to other relevant reported algorithms. The other sections of the paper are arranged as follows: The mathematical formulation of non-convex ELD problem is presented in section 2. The description of the proposed CSA is given in section 3. Section 4 describes how the CSA is applied to the ELD problem. The simulation results, statistical analysis, and comparison results are shown in section 5. Section 6 shows the conclusion of the paper.

\section{PROBLEM FORMULATION}

The non-smooth quadratic cost function is more accurate in the representation of the ELD problem. VPL and piecewise quadratic functions due to MFO are examples of this type of cost functions.

\subsection{Objective function}

ELD with valve-point loading effects: When the steam admission valves are opened to control the output power and to obtain higher power levels from the generation units, a sharp increase in throttling losses occurrs. This causes ripples in the fuel-cost curve [7]. As the valve is progressively lifted, these losses decrease until the valve is completely open. This is known as VPL, which can be mathematically modelled as follows:

$$
F_{i}\left(P_{G i}\right)=\alpha_{i}+\beta_{i} P_{G i}+\gamma_{i} P_{G i}^{2}+\left|\rho_{i} \sin \left(\eta_{i}\left(P_{G i}^{m i n}-P_{G i}\right)\right)\right|
$$

ELD with multiple fuels: Practically, multiple sources of fuel can be used in the thermal power stations to supply the generation units. In this case, the piecewise quadratic cost function will be more suitable in the representation of fuel cost for different fuel types. Hence, the objective of the ELD problem with piecewise fuel cost function is to find the minimum total fuel cost among the available fuels of each unit while satisfying the system constraints [2-4]. This can be mathematically formulated as follows:

$$
F_{i}\left(P_{G i}\right)=\left\{\begin{array}{cc}
\alpha_{i 1}+\beta_{i 1} P_{G i}+\gamma_{i 1} P_{G i}^{2} & P_{G i}^{\min } \leq P_{G i} \leq P_{G i 1} \text { for fuel } 1 \\
\alpha_{i 2}+\beta_{i 2} P_{G i}+\gamma_{i 2} P_{G i}^{2} & P_{G i 1} \leq P_{G i} \leq P_{G i 2} \text { for fuel } 2 \\
& \cdot \\
& \cdot \\
\alpha_{i L}+\beta_{i L} P_{G i}+\gamma_{i L} P_{G i}^{2} & P_{G i L-1} \leq P_{G i} \leq P_{G i}^{\max } \text { for fuel } L
\end{array}\right.
$$

where, $\alpha_{i L}, \beta_{i L}$ and $\gamma_{i L}$ are the cost coefficients of the $i$-th generator for the fuel type $L$.

ELD with multiple fuels and valve-point loading effects: The fuel cost function when VPL and MFO are considered can be represented as follows:

$$
F_{i}\left(P_{G i}\right)=\left\{\begin{array}{ccc}
\alpha_{i 1}+\beta_{i 1} P_{G i}+\gamma_{i 1} P_{G i}^{2}+\left|\rho_{i 1} \sin \left(\eta_{i 1}\left(P_{G i}^{\min }-P_{G i}\right)\right)\right| & P_{G i}^{\min } \leq P_{G i} \leq P_{G i 1} & \text { for fuel } 1 \\
\alpha_{i 2}+\beta_{i 2} P_{G i}+\gamma_{i 2} P_{G i}^{2}+\left|\rho_{i 2} \sin \left(\eta_{i 2}\left(P_{G i}^{m i n}-P_{G i}\right)\right)\right| & P_{G i 1} \leq P_{G i} \leq P_{G i 2} & \text { for fuel } 2 \\
\cdot & \\
\cdot & \\
\alpha_{i L}+\beta_{i L} P_{G i}+\gamma_{i L} P_{G i}^{2}+\left|\rho_{i L} \sin \left(\eta_{i L}\left(P_{G i}^{\min }-P_{G i}\right)\right)\right| & P_{G i L-1} \leq P_{G i} \leq P_{G i}^{\max } & \text { for fuel } L
\end{array}\right.
$$

\subsection{Constraints}

Power balance constraint: The total power generation must satisfy the total load demand $\left(P_{D}\right)$ and the transmission power losses (Ploss) [4]. Hence,

$$
\sum_{i=1}^{N G} P_{G i}=P_{D}+\text { Ploss }
$$

Generation limits constraint: The real output power from each generator must be between its minimum and maximum limits as follows [1]:

$$
P_{G i}^{\min } \leq P_{G i} \leq P_{G i}^{\max }, \quad i=1, \ldots \ldots, N G
$$




\section{CROW SEARCH ALGORITHM}

Crow search algorithm (CSA) is a meta-heuristic optimization algorithm presented by Askarzadeh in 2016 [12]. The main idea of the CSA is obtained from noticing the social behavior of crows, which consider the most intelligent birds. Crows live in the form of flocks. They characterized by having a good memory [13]. Crows are thieves. They watch other birds, including the other crow members on the flock, and observe where they hide their food. Once the other birds leave, they steal their food. The crows use their intelligence to hide their excess food in a hideout spot and restore this food when they need [14]. It is difficult to find the crow stored food. If a crow discovers another one is going after it, it will try to deceive that crow and will go to another position [12]. This intelligent manner of the crows is similar to the optimization process, and CSA attempts to simulate that behavior to find the optimal solutions to the optimization problems [12]. If there is a solution space with dimension $d$ has a crow folk of $n$ crows, then the position $X$ of crow $i$ at iteration $t$ can be expressed by the vector:

$$
X^{i, t}=\left[x_{1}^{i, t}, x_{2}^{i, t}, \ldots \ldots, x_{d}^{i, t}\right]
$$

where:

$i=1: n$,

$t=1: t^{\max }$, and

$t^{\max }$ is the maximum number of iterations.

Each crow has a memory $m^{i, t}$ in which it stores the best position of its storing food source. The vector $X$ contains the random initial positions of the crows. These positions are updated at each iteration, and this process is repeated until the stopping criterion is met. To update the positions of the crows, there are two cases $[12,14]$ :

Case 1: Crow $j$ does not recognize that crow $i$ is going after it; hence, crow $i$ will get close the storing place of crow $j$. In this state, the position of crow $i$ will be updated as follows:

$$
X^{i, t+1}=X^{i, t}+r a_{i} \times f l^{i, t} \times\left(m^{j, t}-X^{i, t}\right)
$$

where:

$r a_{i}$ is a random number with uniform distribution and its value between 0 and 1 .

$f l^{i, t}$ is the flight length of crow $i$ at iteration $t$.

$f l$ has an effect on the capability of the search [12]. Adjusting the value of $f l$ will help in the convergence of the search algorithm [13].

Case 2: Crow $j$ recognizes that crow $i$ is going after it. So, it will move to another position to deceive crow $i$ and to save its food.

The summary of the two cases is as follows:

$$
X^{i, t+1}=\left\{\begin{array}{cc}
X^{i, t}+r a_{i} \times f l^{i, t} \times\left(m^{j, t}-X^{i, t}\right) & r_{j} \geq A P^{j, t} \\
\text { random position } & \text { otherwise }
\end{array}\right.
$$

where $r_{j}$ is the uniform distributed random number in the range of $[0,1]$, and $A P$ is the awareness factor. The value of $A P$ controls the intensification and diversification of the optimization process. Decreasing the value of $A P$ will increase the chance of finding the storing food sources by the crows. This would amplify the intensification of the algorithm [12]. However, increasing the value of $A P$ may make the crows search the space randomly, which decreasing their chance to find the storing food sources. This leads to amplifying the diversification of the algorithm [13]. Pseudo code of the CSA can be described as shown in below.

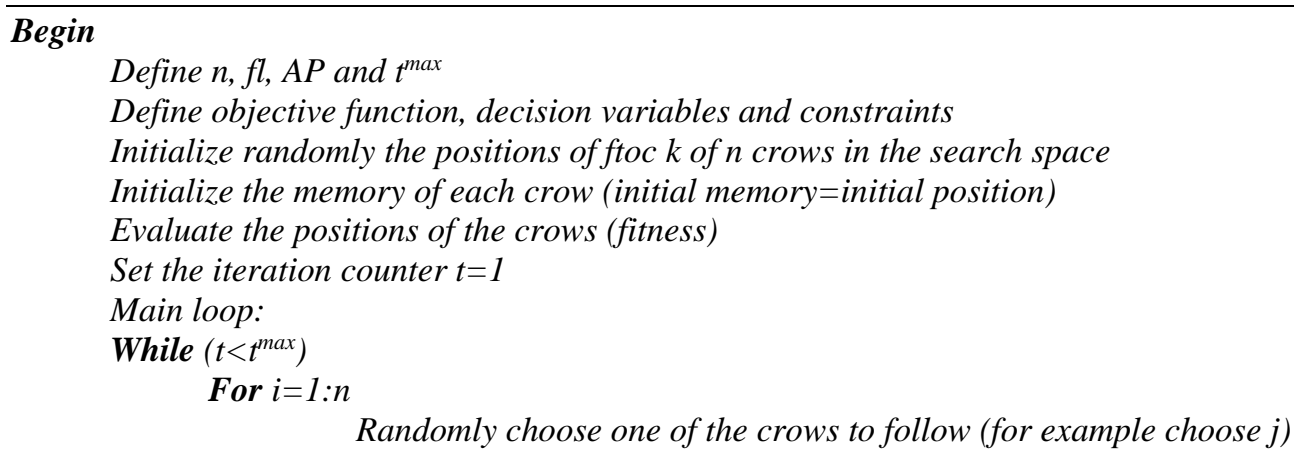




\author{
End for \\ Check the feasibility of new positions \\ Evaluate the new position of the crows \\ Update the memory of crows
}

If $r_{j}>A P^{j t}$

$X^{i, t+1}=X^{i, t}+r a_{i} \times f l^{i, t} \times\left(m^{j, t}-X^{i, t}\right)$

Else

End if

$X^{i, t+1}=a$ random position of search space

End while

End

Find the optiomal solution

\title{
4. APPLICATION OF CSA TO ELD PROBLEM
}

In this paper, the main steps of the proposed CSA implementation to solve the ELD problem can be explained as follows:

Step 1: Define the algorithm parameters including $n, t^{\max }, f l$, and $A P$, and define the system constraints including upper and lower values of power generation units and power balance constraint.

Step 2: Initialization of the position and memory of the crows: Generate randomly the initial population of crow folk positions in the search space using (9) as follows:

$$
X_{i, k}=X_{k(\min )}+\left(X_{k(\max )}-X_{k(\min )}\right) \times \text { rand } \quad i=1: n, k=1: d
$$

where rand is a uniformly distributed random number between 0 and $1 . X_{i, k}$ is a matrix with dimensions $n \times d$. The position of each crow obtained by (9) represents a suggested solution to the ELD problem. The number of control variables $d$ equals the number of committed generation units $(d=N G)$.

Next, generate the crow initial memory. In this work, it is supposed that the initial memory of the crows is the same as their initial positions.

Step 3: Evaluate the objective function and calculate the fitness value for each crow: Calculate the fitness value by substituting the positions into the fuel cost objective function, which is represented by:

1. Equation (1) when VPL is considered or, (1)

2. Equation (2) when MFO is considered or, (2)

3. Equation (3) when MFO and VPL are considered. (3)

Step 4: Generate the new positions of crows: Find the new positions of the crows in the $d$-dimensional search space as follows: If crow $i$ looks for a new position, it will randomly choose one of the crows $j$ and go after it to discover the position of its hidden food sources $\left(m_{j}\right)$. The new position of crow $i$ will be found according to (8).

Step 5: Checking the feasibility of new positions: Check the feasibility of the new position of each crow, and update the position based on it. If the new position is feasible, the position is updated, and if not, the crow remains in its current position and does not move to the new position found.

Step 6: Evaluate the objective function of new positions: Evaluate the new positions, and obtain the new fitness values as explained in step 3.

Step 7: update memory: Update the crows memory as follows [12]:

$$
M^{i, t}=\left\{\begin{array}{lc}
X^{i, t+1} & \text { if } f\left(X^{i, t+1}\right) \text { is better than } f\left(M^{i, t}\right) \\
M^{i, t} & \text { otherwise }
\end{array}\right.
$$

Equation (10) states that if the fitness of new positions is better than the fitness of memory positions, the memory is updated.

Step 8: End the algorithm if the stopping criterion is met: If the maximum number of iterations is reached, End the algorithm.

Step 9: Find the optimal solution: Find the optimal solution which includes the optimal output power of generation units and its corresponding optimal value of total fuel cost. 


\section{SIMULATION RESULTS AND DISCUSSION}

The proposed CSA is implemented in MATLAB 7.10.0 environment. The programs are run on a personal computer with an Intel Core I5, 2.2 GHz processor, 4 GB RAM, and the Windows 8.1 operating system. Due to the random nature of CSA, several trials with different initial populations are carried out to obtain a useful conclusion of the performance of the algorithm and to choose the best values of the proposed CSA important parameters, which include $n, f l$, and $A P$. To optimize these parameters, several experiments are run by varying their values as follows:

$A P$ is changed from 0 to 1 with a step $0.05, f l$ is changed from 0 to 5 with a step 0.1 , and $n$ is changed from 50 to 250 with step 5.The value of one parameter is changed in its range while the other parameters are fixed. For each combination, the ELD problem is solved, and the statistical indices ofthe objective function are calculated. The best values of the parameters which gave the minimum cost are chosen as the optimal settings of control parameters. It is found that the most suitable values for $f l$ and $A P$ for cases 1 and 2 are 2.0 and 0.1 , respectively, and for case 3 are 3.0 and 0.1 .

\subsection{Case 1: ELD with valve-point loading effects}

In this case, the performance of the proposed CSA in solving non-convex ELD with VPL is discussed. The 10-unit test system [15] is adopted for this study. The test system consists of ten generating units with load demand $2000 \mathrm{MW}$. Table 1 shows the system data. The power losses are neglected. $n$ and $t^{\max }$ are selected to be 60 and 10000, respectively. The total execution time of CSA is 36.786 sec, and the execution time per generation is $0.003 \mathrm{sec}$. The power dispatch results are listed in Table 2 along with the min, mean, and max values of fuel cost. From this table, it is observed that the system constraints are satisfied successfully. The optimal value of the cost obtained by the proposed CSA is $10617.0 \$ / \mathrm{hr}$. The effectiveness of the proposed CSA is compared with PSO [15], MSCO [16], and PHOA [17], as given in Table 2. It is clear that the proposed CSA outperforms these methods as it gives the best values of the min and the mean cost compared to the other methods.

Table 1. Limits of generation units and cost coefficients for 10-unit test system

\begin{tabular}{cccccccc}
\hline Unit & $\begin{array}{c}\text { Min } \\
\text { Mw }\end{array}$ & $\begin{array}{c}\text { Max } \\
\text { U1 }\end{array}$ & $\begin{array}{c}\alpha \\
\$ \mathrm{MW}^{2}\end{array}$ & $\begin{array}{c}\beta \\
\$ \mathrm{MW}\end{array}$ & $\begin{array}{c}\gamma \\
\$\end{array}$ & $\begin{array}{c}\eta \\
\mathrm{MW}^{-1}\end{array}$ \\
\hline U1 & 10 & 55 & 0.12951 & 40.5407 & 1000.403 & 33 & 0.0174 \\
$\mathrm{U} 2$ & 20 & 80 & 0.10908 & 39.5804 & 950.606 & 25 & 0.0178 \\
$\mathrm{U} 3$ & 47 & 120 & 0.12511 & 36.5104 & 900.705 & 32 & 0.0162 \\
$\mathrm{U} 4$ & 20 & 130 & 0.12111 & 39.5104 & 800.705 & 30 & 0.0168 \\
U5 & 50 & 160 & 0.15247 & 38.539 & 756.799 & 30 & 0.0148 \\
U6 & 70 & 240 & 0.10587 & 46.1592 & 451.325 & 20 & 0.0163 \\
U7 & 60 & 300 & 0.03546 & 38.3055 & 1243.531 & 20 & 0.0152 \\
U8 & 70 & 340 & 0.02803 & 40.3965 & 1049.998 & 30 & 0.0128 \\
U9 & 135 & 470 & 0.02111 & 36.3278 & 1658.569 & 60 & 0.0136 \\
U10 & 150 & 470 & 0.01799 & 38.2704 & 1356.659 & 40 & 0.0141 \\
\hline
\end{tabular}

Table 2. Simulation results for 10-unit test system

\begin{tabular}{ccccc}
\hline & Proposed CSA & PSO [15] & MSCO [16] & PHOA [17] \\
\hline$P_{1}$ & 55.0000 & 53.1000 & 55.0000 & 55.0000 \\
$P_{2}$ & 80.0000 & 79.2000 & 80.0000 & 80.0000 \\
$P_{3}$ & 89.0818 & 112.000 & 91.4067 & 98.2792 \\
$P_{4}$ & 80.1957 & 121.000 & 73.8654 & 73.2943 \\
$P_{5}$ & 66.3500 & 98.8000 & 70.5700 & 70.2278 \\
$P_{6}$ & 70.0000 & 100.000 & 70.0000 & 72.7025 \\
$P_{7}$ & 290.6553 & 299.000 & 282.6504 & 270.4959 \\
$P_{8}$ & 328.7171 & 320.000 & 340.0000 & 340.0000 \\
$P_{9}$ & 470.0000 & 467.000 & 470.0000 & 470.0000 \\
$P_{10}$ & 470.0000 & 356.000 & 466.5075 & 470.0000 \\
Min. cost & 10617.0 & 107620 & 10619.8 & 10621.0 \\
Mean cost & 10618.0 & - & 10632.0 & 10621.0 \\
Max. cost & 10796.0 & - & 10645.0 & 10621.0 \\
\hline
\end{tabular}

\subsection{Case 2: Piecewise quadratic fuel cost}

The effectiveness of proposed CSA for solving the ELD problem with MFO is studied using the 10unit test system, large-scale test systems [3], and very large-scale test systems [3]. The large-scale and very large-scale test systems are formed based on the 10-unit test system by duplicating it to obtain the required system size. The first test system has 10 units. Each unit can be supplied by two or three different fuels. 
For comparison purposes, the problem is solved for load demands $2400 \mathrm{MW}, 2500 \mathrm{MW}, 2600 \mathrm{MW}$, and $2700 \mathrm{MW}$. The power losses are neglected for all load demands. $t^{\max }$ is selected to be 10000 . The dispatch, the statistical results, and the total execution time are listed in Table 3. The optimal fuel costs obtained by the proposed CSA are $481.7226 \$ / \mathrm{hr}, 526.2388 \$ / \mathrm{hr}, 574.3808 \$ / \mathrm{hr}$, and $623.8092 \$ / \mathrm{hr}$ for the loads $2400 \mathrm{MW}, 2500 \mathrm{MW}, 2600 \mathrm{MW}$, and $2700 \mathrm{MW}$, respectively. Figure 1 shows the convergence characteristic of fuel cost at different load demands. The good performance of proposed CSA in the gradual decrease of the objective function until reaching the minimum value is detected from this figure.

Table 3. Dispatch and statistical results for 10-unit test system with MFO at different load demands

\begin{tabular}{ccccccccc}
\hline & \multicolumn{2}{c}{$2400 \mathrm{MW}$} & \multicolumn{2}{c}{$2500 \mathrm{MW}$} & \multicolumn{2}{c}{$2600 \mathrm{MW}$} & \multicolumn{2}{c}{$2700 \mathrm{MW}$} \\
Unit & Fuel & $P_{i}(\mathrm{MW})$ & Fuel & $P_{i}(\mathrm{MW})$ & Fuel & $P_{i}(\mathrm{MW})$ & Fuel & $P_{i}(\mathrm{MW})$ \\
\hline 1 & 1 & 189.7375 & 2 & 206.5190 & 1 & 189.4093 & 2 & 218.1882 \\
2 & 1 & 202.3375 & 1 & 206.4573 & 1 & 181.3156 & 1 & 211.6625 \\
3 & 1 & 253.8996 & 1 & 265.7391 & 1 & 286.8303 & 1 & 280.7206 \\
4 & 3 & 233.0457 & 3 & 235.9531 & 3 & 210.8350 & 3 & 239.5668 \\
5 & 1 & 241.8340 & 1 & 258.0177 & 1 & 314.8870 & 1 & 278.4753 \\
6 & 3 & 233.0445 & 3 & 235.9531 & 3 & 229.7923 & 3 & 239.5835 \\
7 & 1 & 253.2750 & 1 & 268.8635 & 1 & 289.7424 & 1 & 288.6982 \\
8 & 3 & 233.0436 & 3 & 235.9531 & 3 & 219.1520 & 3 & 239.5767 \\
9 & 1 & 320.3783 & 1 & 331.4877 & 1 & 352.8595 & 3 & 428.4968 \\
10 & 1 & 239.4043 & 1 & 255.0562 & 1 & 325.1766 & 1 & 275.0314 \\
TPG* (MW) & 2400 & 2500 & 2600 & & 2700 \\
Min. cost & 481.7226 & 526.2388 & 574.3808 & 623.8092 \\
Mean cost & 481.8068 & 526.3180 & 574.4136 & 623.8650 \\
Max. cost & 515.8100 & 585.0387 & 599.1933 & 679.6398 \\
Std & 1.3474 & 1.3633 & 0.7535 & 1.2859 \\
Time (sec.) & 10.714 & 10.813 & & 10.552 & & 11.445 \\
\hline
\end{tabular}

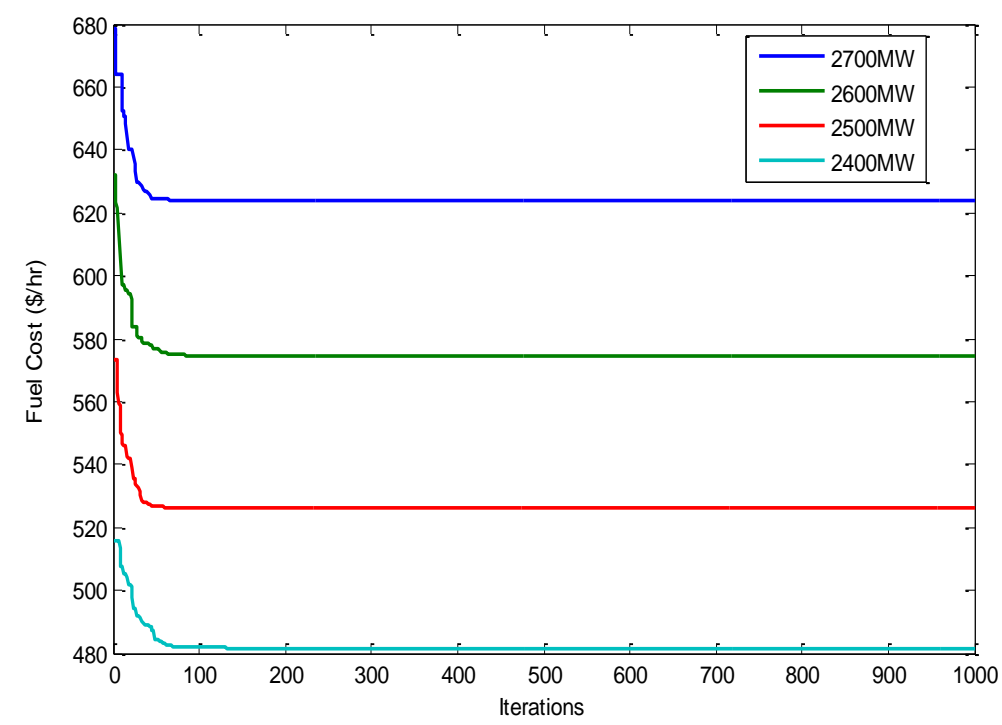

Figure 1. Convergence characteristics of the proposed CSA for 10-unit test system with MFO

The comparison between the optimal fuel cost obtained by the proposed CSA and the other reported algorithms is given in Table 4. For the load demand $2400 \mathrm{MW}$, it is noticed that the proposed CSA gives better fuel cost than ARCGA [7], HNUM [18], and MPSO [19] and it almost obtains the same value of fuel cost compared to other methods. For load demand $2500 \mathrm{MW}$, the proposed CSA obtains the same optimal fuel cost as AHNN [20], and it gives better fuel cost than the other algorithms. For load demand 2700 MW, the proposed CSA obtains the same fuel cost as QP-ALHN [3], RCGA [21], HRCGA [21], and MPSO [19] and it gives better fuel cost compared to the other methods. It should be mentioned that HNUM [18] did not satisfy the power balance constraint for all load demand. 
Table 4. Comparison of the best fuel cost for 10-unit test system with MFO

\begin{tabular}{ccccc}
\hline Method & $2400 \mathrm{MW}$ & $2500 \mathrm{MW}$ & $2600 \mathrm{MW}$ & $2700 \mathrm{MW}$ \\
\hline Proposed CSA & 481.722 & 526.230 & 574.380 & 623.809 \\
QP-ALHN [3] & 481.723 & 526.239 & 574.381 & 623.809 \\
ARCGA [7] & 481.743 & 526.259 & 574.405 & 623.828 \\
AHNN [20] & 481.720 & 526.230 & 574.370 & 626.240 \\
HGA [21] & - & 526.240 & 574.380 & 626.810 \\
RCGA [21] & 481.723 & 526.239 & 574.396 & 623.809 \\
HRCGA [21] & 481.722 & 526.238 & 574.380 & 623.809 \\
AIS [23] & - & 526.240 & 574.380 & 623.810 \\
HNUM [18] & 488.500 & 526.700 & 574.030 & 625.180 \\
MPSO [19] & 481.723 & 526.239 & 574.381 & 623.809 \\
\hline
\end{tabular}

The proposed CSA method is tested on large-scale test systems with 30, 60, and 100 generation units. $t^{\max }$ is selected to be 10000 . The dispatch results for the 60-unit test system with MFO are given in Table 5. The optimal value of fuel cost obtained by the proposed CSA for the 60-unit test system is $3742.9 \$ / \mathrm{hr}$. The results of large-scale test systems with 30,60 , and 100 generation units are compared to QP-ALHN [3], CGA [22], and IGA-AMUM [22]. The comparison results and total execution time are given in Table 6. From this table, we observed that the proposed CSA gives the approximate results as QP-ALHN [3] for all systems, and it gives better results than CGA [22] and IGA-AMUM [22]. The convergence characteristic of fuel cost objective functions for the large-scale systems is shown in Figure 2. Also, the capability of the proposed CSA method is tested for solving very large-scale test systems with 500, 1500, 2000, and 2500 units. The best fuel cost results and the total execution are given in Table 7. From this table, it is observed that with increasing the size of the test system, the proposed CSA gives better values of fuel cost compared to QP-ALHN [3].

Table 5. Dispatch results for 60-unit test system with MFO

\begin{tabular}{cccccccccccc}
\hline Unit & Fuel & $P_{i}(\mathrm{MW})$ & Unit & Fuel & $P_{i}(\mathrm{MW})$ & Unit & Fuel & $P_{i}(\mathrm{MW})$ & Unit & Fuel & $P_{i}(\mathrm{MW})$ \\
\hline 1 & 2 & 218.2489 & 16 & 3 & 239.6314 & 31 & 2 & 218.2485 & 46 & 3 & 239.6321 \\
2 & 1 & 211.6634 & 17 & 1 & 288.5852 & 32 & 1 & 211.6610 & 47 & 1 & 288.5849 \\
3 & 1 & 280.7220 & 18 & 3 & 239.6314 & 33 & 1 & 280.7226 & 48 & 3 & 239.6320 \\
4 & 3 & 239.6316 & 19 & 3 & 428.5343 & 34 & 3 & 239.6315 & 49 & 3 & 428.5193 \\
5 & 1 & 278.4958 & 20 & 1 & 274.8658 & 35 & 1 & 278.4992 & 50 & 1 & 274.8677 \\
6 & 3 & 239.6308 & 21 & 2 & 218.2516 & 36 & 3 & 239.6307 & 51 & 2 & 218.2521 \\
7 & 1 & 288.5850 & 22 & 1 & 211.6643 & 37 & 1 & 288.5843 & 52 & 1 & 211.6635 \\
8 & 3 & 239.6324 & 23 & 1 & 280.7218 & 38 & 3 & 239.6316 & 53 & 1 & 280.7224 \\
9 & 3 & 428.5167 & 24 & 3 & 239.6315 & 39 & 3 & 428.5328 & 54 & 3 & 239.6318 \\
10 & 1 & 274.8691 & 25 & 1 & 278.4937 & 40 & 1 & 274.8681 & 55 & 1 & 278.4961 \\
11 & 2 & 218.2476 & 26 & 3 & 239.6314 & 41 & 2 & 218.2504 & 56 & 3 & 239.6316 \\
12 & 1 & 211.6634 & 27 & 1 & 288.5852 & 42 & 1 & 211.6629 & 57 & 1 & 288.5820 \\
13 & 1 & 280.7230 & 28 & 3 & 239.6316 & 43 & 1 & 280.7233 & 58 & 3 & 239.6313 \\
14 & 3 & 239.6311 & 29 & 3 & 428.5073 & 44 & 3 & 239.6306 & 59 & 3 & 428.5210 \\
15 & 1 & 278.4981 & 30 & 1 & 274.8683 & 45 & 1 & 278.4962 & 60 & 1 & 274.8650 \\
\end{tabular}

Table 6. Comparison of the min. fuel cost for large-scale test system

\begin{tabular}{cccc}
\hline Method & No. of units & Min. total fuel cost & Execution time (sec) \\
\hline Proposed CSA & 30 & 1871.400 & 21.04 \\
& 60 & 3742.900 & 40.55 \\
QP-ALHN [3] & 100 & 6238.100 & 72.57 \\
& 30 & 1871.426 & 0.13 \\
CGA [22] & 60 & 3742.855 & 0.24 \\
& 100 & 6238.092 & 0.43 \\
IGA-AMUM [22] & 30 & 1873.691 & 263.64 \\
& 60 & 3748.761 & 517.88 \\
& 100 & 6251.469 & 873.70 \\
& 60 & 1872.047 & 80.47 \\
& 100 & 3744.722 & 157.19 \\
\hline
\end{tabular}




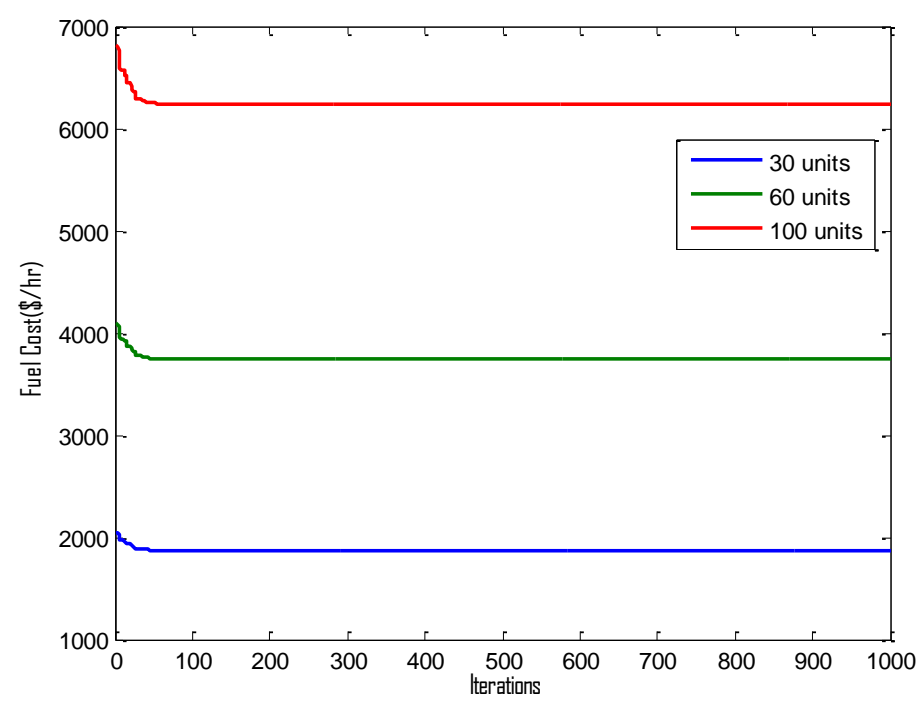

Figure 2. Convergence characteristics of the proposed CSA for large-scale test systems

Table 7. Results of the best fuel cost for very large-scale test system

\begin{tabular}{cccc}
\hline Method & No. of units & Total fuel cost & Execution time (sec) \\
\hline Proposed CSA & 500 & 31191.000 & 320.40 \\
& 1500 & 93572.000 & 950.55 \\
& 2000 & 124760.00 & 1302.20 \\
QP-ALHN [3] & 2500 & 155950.00 & 1500.46 \\
& 500 & 31190.460 & 9.672 \\
& 1500 & 93571.370 & 172.828 \\
& 2000 & 124761.83 & 375.781 \\
& 2500 & 155952.29 & 676.563 \\
\hline
\end{tabular}

\subsection{Case 3: Piecewise quadratic fuel cost with valve-point loading effects}

In this case, the VPL is considered along with MFO. The capability of the proposed CSA to solve this problem is tested on the 10-unit test system with $2700 \mathrm{MW}$ load demand. $t^{\max }$ is selected to be 10000 . The total execution time is $15.70 \mathrm{sec}$. The dispatch results and the comparison results are given in Table 8 . From this table, it is noticed that only proposed CSA, SSA [4], DSD [4], CGA-MU [24], CSA [25], and BSA [26] satisfy power balance constraint, and the other methods HCRO-DE [4], CBPSO-RVM [23], QPSO [27], and NPSO-LRS [27] violate it. Also, it is noticed that the value of min fuel cost is increased from $623.8092 \$ / \mathrm{hr}$ in case 1 for $2700 \mathrm{MW}$ to $623.8342 \$ / \mathrm{hr}$ in this case due to the VPL. The statistical results of the proposed CSA method are compared to IGA-MU [24], CGA-MU [24], and CSA [25], as given in Table 9. It clear that the proposed CSA gives better min and mean values of fuel cost compared to the other methods.

Table 8. Results of 10-unit test system with piecewise quadratic cost function and VPL comparing with other algorithms

\begin{tabular}{cccccccccccc}
\hline Unit & Fuel & $\begin{array}{c}\text { Proposed } \\
\text { CSA }\end{array}$ & SSA [4] & $\begin{array}{c}\text { HCRO- } \\
\text { DE [4] }\end{array}$ & $\begin{array}{c}\text { DSD } \\
{[4]}\end{array}$ & $\begin{array}{c}\text { CBPSO- } \\
\text { RVM } \\
{[23]}\end{array}$ & $\begin{array}{c}\text { CGA- } \\
\text { MU } \\
{[24]}\end{array}$ & $\begin{array}{c}\text { QPSO } \\
{[27]}\end{array}$ & $\begin{array}{c}\text { NPSO- } \\
\text { LRS [27] }\end{array}$ & $\begin{array}{c}\text { CSA } \\
{[25]}\end{array}$ & $\begin{array}{c}\text { BSA } \\
{[26]}\end{array}$ \\
\hline 1 & 2 & 218.8548 & 219.16264 & 213.4589 & 218.59400 & 219.2073 & 222.0108 & 224.7063 & 223.3352 & 219.1817 & 218.57 \\
2 & 1 & 212.4086 & 211.65928 & 209.7300 & 211.71174 & 210.2203 & 211.6352 & 212.3882 & 212.1957 & 211.6596 & 211.21 \\
3 & 1 & 281.5418 & 280.68427 & 332.0143 & 280.65706 & 278.5456 & 283.9455 & 283.4405 & 276.2167 & 280.6571 & 279.56 \\
4 & 3 & 239.0244 & 239.95493 & 237.7581 & 239.63943 & 276.4120 & 237.8052 & 289.6530 & 286.0163 & 239.9551 & 239.50 \\
5 & 1 & 280.1966 & 276.38750 & 269.1476 & 279.93452 & 274.6470 & 280.4480 & 283.8190 & 286.0163 & 276.4164 & 279.97 \\
6 & 3 & 239.6657 & 239.79532 & 238.9677 & 239.63943 & 240.5797 & 236.0330 & 241.0024 & 239.7974 & 239.7953 & 241.11 \\
7 & 1 & 287.4733 & 290.07417 & 292.3267 & 287.72749 & 285.5388 & 292.0499 & 287.8571 & 291.1767 & 290.0985 & 289.79 \\
8 & 3 & 239.9521 & 239.82117 & 237.7557 & 239.63943 & 240.6323 & 241.9708 & 240.6245 & 241.4398 & 239.8207 & 240.57 \\
9 & 3 & 426.0197 & 426.37501 & 413.6294 & 426.58829 & 429.4008 & 424.2011 & 407.9870 & 429.2637 & 426.3626 & 426.88 \\
10 & 1 & 274.8632 & 276.08571 & 266.3841 & 275.86861 & 276.1815 & 269.9005 & 278.2120 & 278.9541 & 276.0531 & 272.79 \\
TPG & & 2700.00 & 2700.00 & 2711.1725 & 2700.00 & 2731.365 & 2700.00 & 2749.69 & 2764.4119 & 2700.00 & 2700.00 \\
Fuel & & 623.8342 & 623.6433 & 628.9605 & 623.8265 & 624.3911 & 624.7193 & 624.1505 & 623.9258 & 623.8361 & 623.9016 \\
cost & & & & & & & & & & \\
\hline
\end{tabular}


Table 9. Statistical results for 10-unit test system with MFO and VPL

\begin{tabular}{ccccc}
\hline Method & Min fuel cost & Mean fuel cost & Max fuel cost & Std \\
\hline Proposed CSA & 623.8342 & 623.8566 & 680.0601 & 0.6290 \\
CGA-MU [24] & 624.7193 & 627.6087 & 633.8652 & - \\
IGA-MU [24] & 624.5178 & 625.8692 & 630.8705 & - \\
CSA [25] & 623.8361 & 623.9626 & 624.8304 & 0.0116 \\
\hline
\end{tabular}

\section{CONCLUSION}

In this paper, the CSA method has been successfully implemented to solve the non-convex practical ELD problem with valve-point loading effects and multi-fuel options. The 10-unit test system has been considered. In addition to large-scale test systems with 30-unit, 60-unit, and 100-unit, and very large-scale test systems with 500, 1500, 2000, and 2500 units. Three different cases are efficiently studied. The simulation results confirm the robustness and effectiveness of the proposed CSA method to solve the practical ELD problem with different formulations. Good convergence characteristics of the CSA method is detected. The simulation results are compared to the reported algorithms. The comparison of results and the statistical analysis confirm the effectiveness, high-quality solutions, and superiority of the proposed CSA for solving the practical ELD problem.

\section{REFERENCES}

[1] R. Habachi, et al., "Economic and emission dispatch using cuckoo search algorithm," International Journal of Electrical and Computer Engineering (IJECE), vol. 9, no. 5, pp. 3384-3390, Oct. 2019.

[2] Y. V. K. Reddy and M. D. Reddy, "Grey Wolf Optimization for Solving Economic Dispatch with Multiple Fuels and Valve Point Loading," International Journal of Information Engineering and Electronic Business, vol. 1, pp. 50-57, 2019.

[3] Vo N. Dieu and P. Schegner, "Augmented Lagrange Hopfield network initialized by quadratic programming for economic dispatch with piecewise quadratic cost functions and prohibited zones," Applied Soft Computing, vol. 13, pp. 292-301, 2013.

[4] J. J.Q. Yu and V. O. K. Li, "A Social Spider Algorithm for Solving the Non-convex Economic Load Dispatch Problem," Neurocomputing, Jul. 2015.

[5] Ld. S. Coelho and V. C. Mariani, "Combining of chaotic differential evolution and quadratic programming for economic dispatch optimization with valve-point effect," IEEE Transactions on Power Systems, vol. 21, no. 2, pp. 989 - 996, 2006.

[6] J. K. Delson and S. M. Shahidehpour, "Linear programming applications to power system economics, planning and operations," IEEE Transactions on Power Systems, vol. 7, no. 3, pp. 1155-1163, Aug. 1992.

[7] N. Amjady and H. Nasiri-Rad, "Solution of nonconvex and nonsmooth economic dispatch by a new adaptive real coded genetic algorithm," Expert Systems with Applications, vol. 37, pp. 5239-5245, 2010.

[8] B. R. Adarsh, et al. "Economic dispatch using chaotic bat algorithm," Energy, vol. 96, pp. 666-675, 2016.

[9] B. Bentouati, et al., "Elephant Herding Optimization for Solving Non-convex Optimal Power Flow Problem," Journal of Electrical and Electronics Engineering, vol. 10, no. 1, pp. 31-40, 2017.

[10] M. Modiri-Delshad and N. Abd Rahim, "Multi-objective backtracking search algorithm for economic emission dispatch problem," Applied Soft Computing, vol. 40, pp. 479-494, 2016.

[11] A. A. Elsakaan, et al., "Economic Power Dispatch with Emission Constraint and Valve Point Loading Effect Using Moth Flame Optimization Algorithm," Advanced Engineering Forum, vol. 28, pp. 139-149, Jun. 2018.

[12] A. Askarzadeh, "A novel metaheuristic method for solving constrained engineering optimization problems: Crow search algorithm," Computers and Structures, vol. 169, pp. 1-12, 2016.

[13] A. A. Abou El Ela, et al., "Application of the Crow Search Algorithm for Economic Environmental Dispatch," 2017 Nineteenth International Middle east power Systems Conference (MEPCON), Menoufia University, Egypt, Dec. 2017.

[14] B. Z. Dr-Asli, et al., "Chapter 14: Crow Search Algorithm (CSA)," Advanced Optimization by Nature-Inspired Algorithms, Jul. 2017

[15] S P. S. Bhullar and J. K. Dhami, "Particle Swarm Optimization Based Economic Load Dispatch with Valve Point Loading," International Journal of Engineering Research \& Technology (IJERT), vol. 4, no. 5, 2015.

[16] R. M. Rizk-Allah, et al., "A New Sine Cosine Optimization Algorithm for Solving Combined Non-Convex Economic and Emission Power Dispatch Problems," International Journal on Energy Conversion (I.R.E.CON.), vol. 5, no. 6, Nov. 2017.

[17] R. M. Rizk-Allah, et al., "A novel Parallel hurricane optimization algorithm for secure emission/economic load dispatch solution," Applied Soft Computing, vol. 63, pp. 206-222, 2018.

[18] C. E. Lin and G. L. Viviani, "Hierarchical economic dispatch for piecewise quadratic cost functions," IEEE Transactions on Power Apparatus and Systems PAS-103, pp. 1170-1175, 1984.

[19] J.-B.Park, et al., "A Particle Swarm Optimization for Economic Dispatch with Nonsmooth Cost Functions," IEEE Transactions on Power Systems, vol. 20, no. 1, 2005.

[20] K.Y. Lee, et al., "Adaptive Hopfield neural networks for economic load dispatch," IEEE Transactions on Power Systems, vol. 13, pp. 519-526, 1998. 
[21] S. Baskar, et al., "Hybrid genetic algorithm solution to economic dispatch problem," International Journal Computers Electrical Engineering, vol. 39, no. 3, pp. 407-419, May 2003.

[22] C. L. Chiang and C. T. Su, "Adaptive-improved genetic algorithm for the economic dispatch of units with multiple fuel options," Cybernetics and Systems, vol. 36, pp. 687-704, 2005.

[23] C. L. Chiang and C. T. Su, "Adaptive-improved genetic algorithm for the economic dispatch of units with multiple fuel options," Cybernetics and Systems, vol. 36, pp. 687-704, 2005.

[24] C.-L. Chiang, "Improved Genetic Algorithm for Power Economic Dispatch of Units with Valve-Point Effects and Multiple Fuels," IEEE Transactions on Power Systems, vol. 20, no. 4, pp. 1690-1699, Nov. 2005.

[25] F. Mohammadi, H. Abdi, "A modified crow search algorithm (MCSA) for solving load dispatch problem," Applied Soft Computing, vol. 71, pp. 51-65, 2018.

[26] M. Modiri-Delshad and S. Hr. A. Kaboli, "Backtracking search algorithm for solving economic dispatch problems with valve-point effects and multiple fuel options," Energy, vol. 116, pp. 637-649, 2016.

[27] AI Selvakumar and K. Thanushkodi, "A new particle swarm optimization solution to nonconvex economic dispatch problems," IEEE Transaction on Power System, vol. 22, no. 1, pp. 42-51, 2007. 